\title{
Study of contributions of diffractive processes to forward neutral particle pro- duction with the ATLAS-LHCf detector
}

\author{
Qi-Dong Zhou ${ }^{1, *}$ for "the LHCf and ATLAS Collaborations" \\ ${ }^{1}$ Institute for Space Earth Environmental Research, Nagoya University, Furo-cho, Chikusa-ku, Nagoya 464-8601, Japan
}

\begin{abstract}
LHCf is an experiment dedicated to verify the hadronic interaction models by measuring the forward neutral particle production at the LHC. Corresponding data are very important for understanding hadronic interactions occurring in air-shower development. According to the current LHCf results, no simulation model predicts the LHCf data perfectly. In order to provide more specific data, it is necessary to classify the LHCf observables into specific interaction types: diffraction or non-diffraction. Combining the information of ATLAS, LHCf is able to classify these specific interaction types experimentally. Especially, the ATLAS-LHCf joint experiment will have the unique sensitivity to low mass diffraction. LHCf and ATLAS have succeeded in the common data-taking in $p-p$ collisions at $\sqrt{s}=13 \mathrm{TeV}$. We will report the first ATLAS-LHCf joint analysis result and discuss the impact of the corresponding joint analysis result to the determination of mass composition of ultra-high energy cosmic-rays.
\end{abstract}

\section{Introduction}

The Large Hadron Collider forward (LHCf) experiment [1] is capable of measuring neutral particle productions at zero degree of the LHC. Corresponding particles are known to play an important role in controlling the airshower profile. In particular, the LHC allows to provide a unique chance to access hadronic interactions at $10^{17}$ $\mathrm{eV}$ as the laboratory equivalent collision energy. LHCf had a common data acquisition with ATLAS experiment in the operation at $\sqrt{s}=13 \mathrm{TeV} p$ - $p$ collisions. ATLAS [2] and LHCf [1] detectors are located at the same Interaction Point of the LHC, while having different pesudorapidity coverage. LHCf covers the very forward region and ATLAS has the sensitivity to the central region. The ATLAS central information makes it possible to classify the LHCf observed events into diffraction or non-diffraction on an event-by-event basis.

\section{Diffractive dissociation}

In proton-proton interactions, events can be classified into either elastic or inelastic; inelastic collisions can be furthermore categorized into diffractive or non-diffractive processes as shown in the top box of Fig. 1. In general, the approaches to implement the diffractive and nondiffractive processes are totally different in the hadronic interaction models. The top pad of Fig. 2 shows the diffractive mass distribution as a function $\log _{10}\left(\xi_{X}\right)$, where $\xi_{X}=M_{X}^{2} / s$ and $M_{X}$ is the invariant mass of the X-system (refer to Fig. 1). It is still poorly constrained on the aspect of diffraction in the models due to a lack of experimental

\footnotetext{
*e-mail: zhouqidong@isee.nagoya-u.ac.jp
}

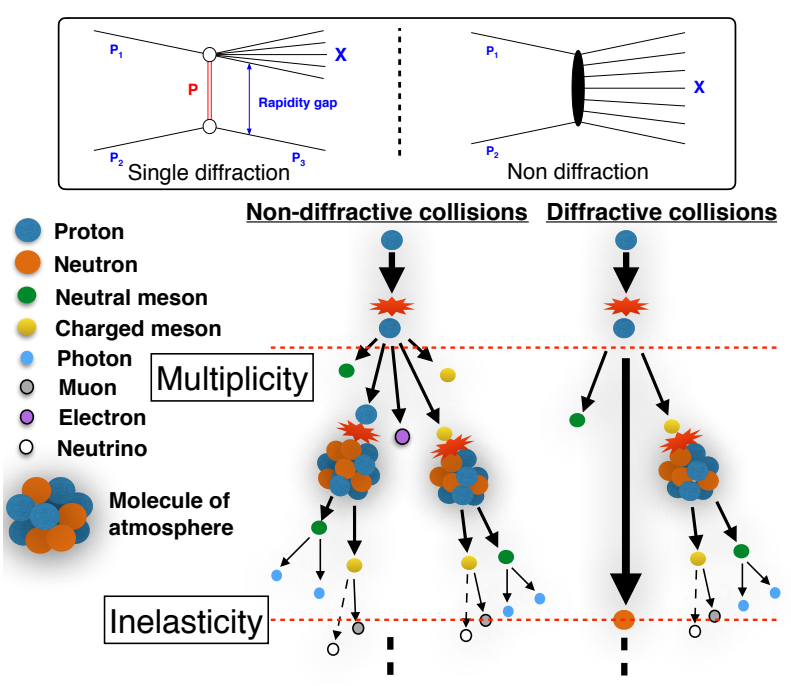

Figure 1. The Feynman diagrams of Single Diffraction (SD, $p p \rightarrow p X$ ) and Non-Diffraction (ND, $p p \rightarrow X$ ) in the box. Image of air-shower development in the atmosphere (bottom pad). The interaction between a primary cosmic-ray and the atmosphere will have a significantly different air-shower profile when the collision type is different: diffraction or non-diffraction. It will directly impact on the parameters of multiplicity and inelasticity which will affect the determination of $X_{\max }$.

data. Therefore, there is a large discrepancy between the models.

There is an operational characteristic of diffractive interactions which is a large angle separation among the final state systems so called rapidity gap $\Delta \eta$ as the Feynman diagram illustrated in the top box of Fig. 1. The size and 

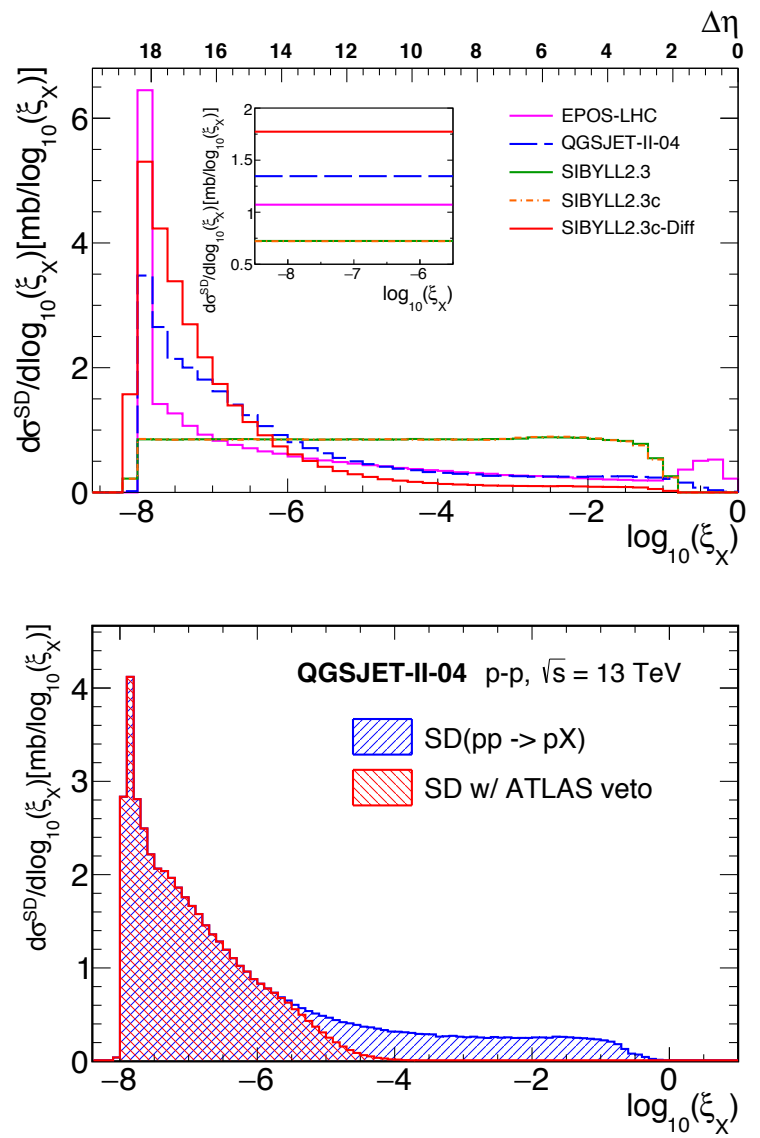

Figure 2. The $\mathrm{SD}(p p \rightarrow p X$; blue) cross section as a function of $\log _{10} \xi_{X}$ predicted by MC simulation models. There is a large discrepancy among models, especially in the low-mass diffraction region (top pad). On the bottom pad, the survived events are mostly low-mass diffraction by applying the ATLAS-veto selection (red) compare to the inclusive SD cross section predicted by QGSJET-II-04 (blue) [3].

location of $\Delta \eta$ in pseudorapidity phase space can be used to identify the collision type. Methodology of identification of diffraction based on the rapidity gap technique has been studied using the ATLAS-LHCf apparatus [3]. As shown in the top pad of Fig. 2, low-mass diffraction is still not well implemented in the models due to the lack of direct measurement data at high energies. It was confirmed that the ATLAS-LHCf joint analysis has a unique sensitivity to such low-mass diffraction region at $\log _{10}\left(\xi_{X}\right)<-5$ as shown in bottom pad of Fig. 2 [3].

The constraint on the aspect of diffraction will directly help for the precise determination of the inelasticity of hadronic interactions. For instance, in the Single Diffractive (SD) case, $\Delta \eta \simeq-\ln \left(\xi_{X}\right)$ and inelasticity $K_{\text {inel }} \simeq \exp (-\Delta \eta)$ [4]. As illustrated in Fig. 1, diffractive and non-diffractive collisions will have very different multiplicity and inelasticity which are important parameters to determine $X_{\max }$ and muon number in the air-shower [5].

\section{ATLAS-LHCf joint analysis}

The first ATLAS-LHCf joint analysis has been accomplished based on $0.191 \mathrm{nb}^{-1}$ of $p$ - $p$ collision data recorded
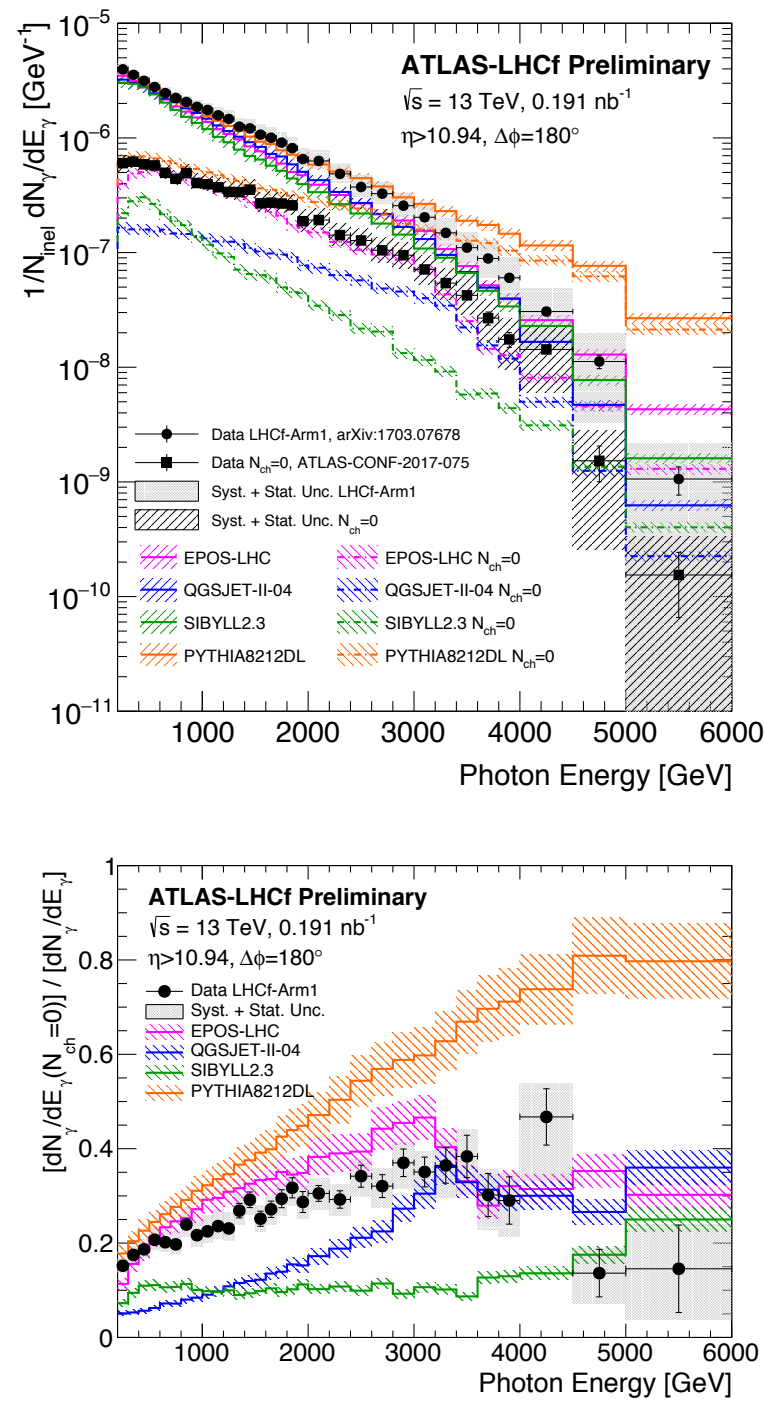

Figure 3. Forward photon energy spectrum measured by the LHCf-Arm1 detector at $|\eta|>10.94$ (top pad). Filled circles show the inclusive-photon spectrum measured in Ref. [6]. Filled squares indicate the spectrum for $N_{\mathrm{ch}}=0$ events [7], where no extra charged particles with $p_{\mathrm{T}}>100 \mathrm{MeV}$ and $|\eta|<2.5$ are present. Colored lines indicate model predictions with (dashed lines) and without (solid lines) the $N_{\mathrm{ch}}=0$ requirement. Ratios of $N_{\mathrm{ch}}=0$ events to the inclusive events as a function of photon energy are shown in the bottom pad.

at $\sqrt{s}=13 \mathrm{TeV}$. The data analysis is based on photon reconstruction in the LHCf-Arm1 detector, as well as on the inner tracking system of the ATLAS detector. The photon energy spectra were measured in two pseudorapidity ranges, $\eta>10.94$ and $8.81<\eta<8.99$, for events with no extra charged particles having $p_{\mathrm{T}}>100 \mathrm{MeV}$ and $|\eta|<2.5$, corresponding to the spectra at $\eta>10.94$ shown with filled squares in the top pad of Fig. 3. Another spectrum shown with filled circles is the inclusive photon spectrum measured by the LHCf-Arm1 detector. The ratio of photon spectrum derived from the low-mass diffraction $\left(N_{\mathrm{ch}}=0\right)$ to the inclusive photon spectrum is shown in the bottom pad of Fig. 3. Both the spectra and ratio re- 

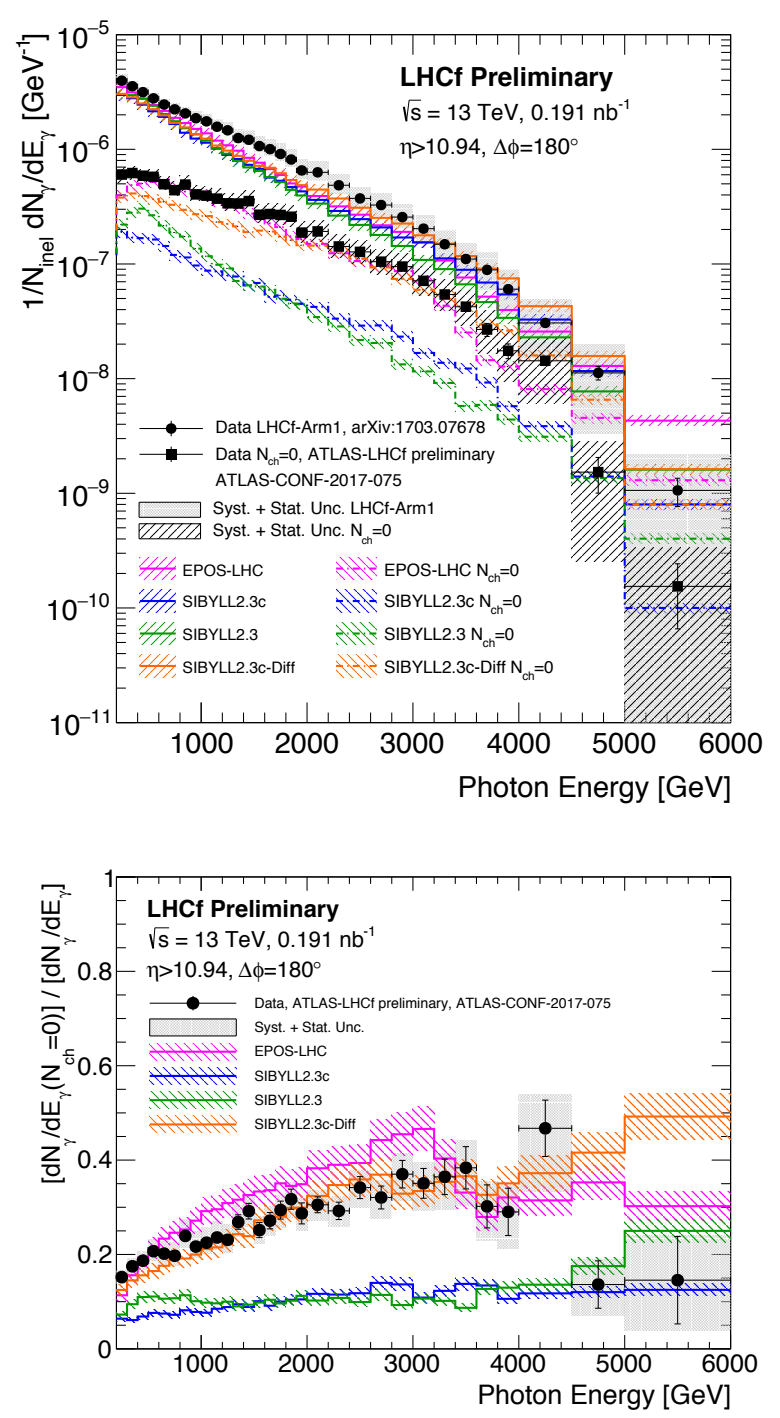

Figure 4. Forward photon energy spectrum measured by the LHCf-Arm1 detector at $|\eta|>10.94$ (top pad). Filled circles show the inclusive-photon spectrum measured in Ref. [6]. Filled squares indicate the spectrum for $N_{\mathrm{ch}}=0$ events [7], where no extra charged particles with $p_{\mathrm{T}}>100 \mathrm{MeV}$ and $|\eta|<2.5$ are present. Colored lines indicate model predictions with (dashed lines) and without (solid lines) the $N_{\mathrm{ch}}=0$ requirement. Ratios of $N_{\mathrm{ch}}=0$ events to the inclusive events as a function of photon energy are shown in the bottom pad. In is different from Fig. 3; predictions of the SIBYLL2.3c model and its modified model, SIBYLL2.3c-Diff are shown in this figure.

sult are compared to the predictions from several hadronic interaction models: EPOS-LHC [8], QGSJET-II-04 [9], SYBILL 2.3 [10, 11], and PYTHIA 8212DL [12, 13]. For general comparison, the EPOS-LHC model shows the best agreement with the data. The ratio of low mass diffraction to the inclusive photon spectra increases from 0.15 to 0.4 at $|\eta|>10.94$, while it keeps constant at 0.15 for $8.81<\eta<8.99$ (corresponding result summarised in [7]). For photon energies above $2 \mathrm{TeV}$, PYTHIA 8 predicts a significantly higher ratio than the one observed in the data. This indicates that the large discrepancy between
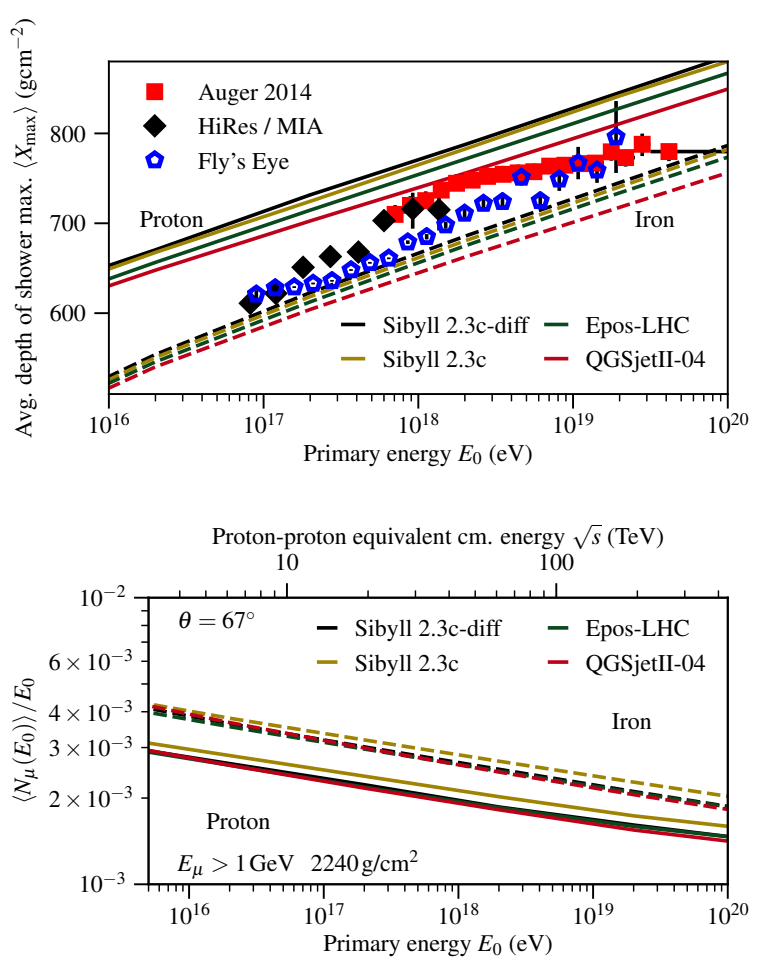

Figure 5. Model predictions of $X_{\max }$ compared with data, including the tuned SIBYLL2.3c-diff model (top). Comparison of muon number at ground level predicted by models, the muon number of tuned SIBYLL2.3c-diff model decreased 8-10\% compared to the original SIBYLL2.3c model [14].

PYTHIA 8 and data in the high-energy photon region reported in Ref. [6] can be due to an overestimation of the diffractive dissociation process in PYTHIA 8. The QGSJET-II-04 and SYBILL 2.3 models predict an average value of the ratio that is much lower than the data observed in the region of $\eta>10.94$ and $8.81<\eta<8.99$ [7]. This suggests that QGSJET-II-04 and SYBILL 2.3 predict too small a contribution of low-mass diffractive events to the forward photon energy spectrum.

\section{Impact to the air-shower observation}

A modification of diffractive mass distribution (pomeron flux) of SIBYLL2.3c was applied according the ATLASLHCf joint analysis result [14]. As shown in the top pad of Fig. 2, the modified SIBYLL2.3c model (SIBYLL2.3cDiff) increased the low mass diffraction. SIBYLL2.3cDiff exhibits a totally different shape of diffractive mass distribution compare to its previous models. In particular, it predicts the largest SD cross-section in the lowmass diffraction regions of $-8.5<\log _{10}\left(\xi_{X}\right)<-5.5$, increasing by $145.7 \%$ compared to SIBYLL2.3 (e.g. 4.336 $\mathrm{mb} \rightarrow 10.654 \mathrm{mb}$ ). Figure 4 shows the comparison of the modified SIBYLL2.3 model with the joint analysis data. By comparing with SIBYLL2.3 and SIBYLL2.3c, SIBYLL2.3c-Diff gives a good agreement with the data, which is comparable to EPOS-LHC. Similarly, as the ratio results shown in bottom pad of Fig. 4, SIBYLL2.3c-Diff is 
dramatically improved and gives the best agreement with the data among the models, even better than EPOS-LHC. Comparisons of the joint analysis result with the prediction of the new tuned SIBYLL2.3c-diff model are shown in Fig. 5. As described in section 2, corresponding tuning of the diffractive mass distribution will directly affect the inelasticity, then the determination of $X_{\max }$. The impact to the determination of $X_{\max }$ has been confirmed by the air shower simulation. As shown in Fig. 5, the tuning shifts $X_{\max }$ by approximately $5 \mathrm{~g} / \mathrm{cm}^{2}$ deeper in the atmosphere. Besides, SIBYLL2.3c-diff predicts a lower number of muons compare to SIBYLL2.3c by approximately 8\%; it moves closer to EPOS-LHC and QGSJET-II-04.

\section{Conclusions}

We accomplished the first joint analysis under the ATLAS and LHCf collaborations based on $0.191 \mathrm{nb}^{-1}$ of $p-p$ collision data recorded at $\sqrt{s}=13 \mathrm{TeV}$. The contribution of low-mass diffractive processes to forward photon production was measured in two pseudorapidity ranges, $\eta>10.94$ and $8.81<\eta<8.99$, with a selection of the events with no extra charged particles having $p_{\mathrm{T}}>100 \mathrm{MeV}$ and $|\eta|<2.5$ based on the ATLAS-LHCf joint analysis. The ratio of the low mass diffractive photon spectrum to the inclusive photon spectrum increases from 0.15 to 0.4 with increasing photon energy up to $4 \mathrm{TeV}$ at $\eta>10.94$, whereas it is found to be relatively constant (around 0.15 ) for $8.81<\eta<8.99$. EPOS-LHC model shows good agreement with the data on both spectrum and ratio results. The QGSJET-II-04 and SYBILL 2.3 models predict too small contribution of lowmass diffraction to the forward photon production in both $\eta>10.94$ and $8.81<\eta<8.99$ regions. SIBYLL2.3c model was improved according to the ATLAS-LHCf joint analysis result by tuning the diffractive mass distribution. The tuned model, SIBYLL2.3c-diff, shifts $X_{\max }$ by approximately $5 \mathrm{~g} / \mathrm{cm}^{2}$ deeper in the atmosphere and decreases the number of muon at ground level by approximately 8 $10 \%$ compare to SIBYLL2.3c.

\section{References}

[1] LHCf Collaboration, JINST, 3, S08006 (2008)

[2] ATLAS Collaboration, JINST, 3, S08003 (2008)

[3] Qi-Dong Zhou et al. Eur. Phys. J. C 77212 (2017)

[4] S. Ostapchenko, Phys. Rev. D 89, 074009 (2014)

[5] R. Ulrich, R. Engel, and M. Unger, Phys. Rev. D 83, 054026 (2011)

[6] LHCf Collaboration, Phys. Lett. B 780, 233-239 (2018)

[7] The ATLAS and LHCf Collaborations, ATLASCONF-2017-075 (2017)

[8] T. Pierog et al., Phys. Rev. C 92, 034906 (2015)

[9] S. Ostapchenko, Phys. Rev. D 83, 014018 (2011)

[10] E.-J. Ahn, R. Engel, T. K. Gaisser, P. Lipari, and T. Stanev, Phys. Rev. D 80, 094003 (2009)

[11] R. Engel, T. K. Gaisser, F. Riehn, and T. Stanev, Proc. 34th Int. Cosmic Ray Conf., The Hague (The Netherlands), 1313 (2015)

[12] T. Sjöstrand, S. Mrenna, and P. Skands, JHEP 05, 026 (2006)

[13] T. Sjöstrand, S. Mrenna, and P. Skands, Comput. Phys. Commun. 178, 852 (2008)

[14] F. Riehn, Private communication. 